\title{
Dexamethasone Sustained-Release Intracanalicular Insert for Control of Postoperative Inflammation After Pars Plana Vitrectomy
}

\section{Ivan J Suñer (D) \\ Marc C Peden}

Retina Associates of Florida, Tampa, FL, USA
Correspondence: Ivan J Suñer Retina Associates of Florida, 602 South MacDill Avenue, Tampa, FL, 33609, USA Email ivansuner@gmail.com
Purpose: To evaluate the safety and efficacy of a dexamethasone sustained-release intracanalicular insert (DII) (Dextenza ${ }^{\circledR}$ Ocular Therapeutix, Inc., Bedford, MA) for control of inflammation and pain after pars plana vitrectomy (PPV) compared to standard topical steroid therapy.

Methods: Retrospective, case-matched comparison of consecutive patients undergoing PPV. Control patients were matched by diagnosis and procedure performed. The primary outcome was the proportion of patients with complete anterior chamber cell clearance (ACCC) at postoperative day 7. Secondary outcomes included proportion developing intraocular pressure (IOP) $>25 \mathrm{mmHg}$, change in mean optical coherence tomography central foveal thickness (OCT CFT), and proportion developing cystoid macular edema (CME) on qualitative analysis of OCT. Results: The DII group had a statistically significant higher rate of complete ACCC compared to the topical steroid group ( $65 \%$ versus $35 \%$, respectively, with $\mathrm{p}=0.01)$. No eyes had IOP $>25$ $\mathrm{mmHg}$ in the DII, compared to 2 eyes in the topical steroid group (not statistically significant). Overall, mean OCT CFT decreased in both groups; one patient had CME in the DII group, as compared to three in the topical steroid group (not statistically significant).

Conclusion: The dexamethasone intracanalicular insert provided excellent safety and efficacy in control of postoperative inflammation following PPV in this retrospective casematched study.

Keywords: dexamethasone intracanalicular insert, sustained-release drug delivery, pars plana vitrectomy, postoperative inflammation, PPV

\section{Introduction}

Pars plana vitrectomy (PPV) with tamponade — whether with air, gas, or silicone oil — is the primary approach for the surgical management of vitreoretinal diseases. ${ }^{1}$ As PPV has evolved to sutureless, small-gauge techniques, ${ }^{2,3}$ more rapid postoperative recovery and decrease in postoperative inflammation following surgery has been observed. ${ }^{4}$

The major obstacle to optimal control of postoperative inflammation after PPV is patient compliance with postoperative regimens. Compliance with topical medications presents a particular challenge since instilling topical agents results in interruption of postoperative positioning. Commonly, multiple topical agents on different schedules are required. This may be especially challenging in patients with decreased vision. Furthermore, instilling eye drops may also present a challenge in patients with lack of experience in topical eyedrop administration ${ }^{5}$ or motor or cognitive issues, which are more prevalent in our elderly population. ${ }^{6}$ 
Physician-administered sustained-release antiinflammatory agents following ocular surgery offer multiple advantages over patient self-administration of topical therapy. Chief among these is improved adherence, which is guaranteed in eyes by virtue of the sustained-delivery platform. Following phacoemulsification, half of patients took fewer than half of their prescribed topical antiinflammatory and antimicrobial doses and only 5\% took $75 \%$ or more of doses. ${ }^{7}$ Nonadherence is closely related to regimen complexity, ${ }^{8}$ which is higher for postoperative patients with multiple medications, multiple drops per day, and a frequently changing number of daily drops associated with steroid tapering.

Recently, a sustained-release dexamethasone intracanalicular insert (DII) has been approved for the short-term control of pain and inflammation following ocular surgery (Dextenza, Ocular Therapeutix, Bedford, MA). ${ }^{9,10}$ The DII is a rod-shaped polyethylene glycol hydrogel insert incorporating $0.4 \mathrm{mg}$ of preservative-free dexamethasone. Fluorescein is conjugated to the hydrogel to facilitate in situ visualization. After punctal dilation, the DII is implanted through the punctum into the canaliculus where, upon hydration, it swells and anchors into place. It dissolves slowly to deliver a sustained and tapered concentration of dexamethasone for up to 30 days. The insert can be placed in the office or the operating room, providing flexibility for administration preoperatively, intraoperatively, or postoperatively at the surgeon's discretion. This implant has been utilized primarily for postoperative treatment following cataract surgery.

Given the positive results in dropless cataract surgery with this implant, we decided to explore a similar dropless steroid regimen for vitrectomy surgery. This would have the advantages of eliminating compliance issues with multiple topical agents, avoiding issues with intravitreal/intracameral agents, and allowing patients to focus on compliance with postoperative positioning regimens. In this retrospective case-matched series, we studied the effectiveness of the dexamethasone intracanalicular insert for control of postoperative pain and inflammation in eyes undergoing PPV with air or gas tamponade.

\section{Materials and Methods Study Design}

This was a retrospective case-matched analysis of consecutive patients undergoing PPV with air or gas tamponade. The study protocol was reviewed and approved by the
Western Investigational Review Board (Puyallup, WA). Patient consent to review medical records was waived by the Western IRB given the retrospective nature of this study. All data accessed for the study was de-identified to protect patient confidentiality and privacy. This study is compliant with the Declaration of Helsinki.

The test group included 23 consecutive patients aged 18 years and older who underwent PPV with air or gas tamponade between August 2019 and October 2019 who received the dexamethasone intracanalicular insert for control of postoperative pain and inflammation. Patients undergoing PPV combined with cataract surgery were included. One eye per patient was included for analysis. This represented all patients undergoing vitrectomy procedures. The control group was comprised of 23 consecutive patients undergoing surgery between January 2019 and July 2019 that were matched to the test group by diagnosis and surgical procedure via the Electronic Health Record (EHR).

The EHR was reviewed for data collection at the baseline preoperative visit (Day 0), postoperative days 1, 7, and 30. This included: demographic information, diagnosis, procedure performed, best-corrected visual acuity (BCVA), intraocular pressure (IOP), presence or absence of anterior chamber cells (ACC) by slit-lamp examination using a narrow beam, bright light setting, and high magnification, evaluation of optical coherence tomography (OCT) central foveal thickness (CFT), and qualitative assessment of OCT for cystoid macular edema (CME) using the Zeiss Cirrus High-Definition Spectral Domain OCT platform. As patients with macular holes did not have a baseline OCT CFT measurement, they were excluded from analysis of change of OCT CFT, but included for analysis of final CFT and development of CME. Adverse events through 30 days of postoperative follow-up were also recorded.

The primary outcome of this study was the proportion of patients with complete anterior chamber cell clearance (ACCC) at one week. Secondary outcomes included the mean change in IOP, proportion developing IOP $>25$ $\mathrm{mmHg}$ at any time point, mean change in OCT CFT at Day 30, and proportion with macular edema on qualitative OCT imaging at Day 30.

\section{Surgical Procedure}

All patients underwent standard PPV surgery by a single surgeon (IJS). 25-gauge sutureless vitrectomy with valved cannulas and air or sulfur hexafluoride $\left(\mathrm{SF}_{6}\right)$ gas 
tamponade was utilized in all patients. In select patients, combined cataract surgery and PPV was performed. The dexamethasone intracanalicular insert was placed in the inferior punctum of the operated eye, as per the product's instructions for use. The insert was positioned so that its proximal tip lay just below the level of the punctal opening. Control patients received standard topical prednisolone acetate $1 \%$ ophthalmic suspension four times per day for the first week, which was tapered over the first month. Patients received topical moxifloxacin $0.5 \%$ ophthalmic solution four times per day for one week for antibiotic prophylaxis.

\section{Analysis}

Descriptive statistics were employed for reporting of demographic data. Primary outcome of complete ACCC was compared using chi-square analysis with p-value $\leq$ 0.05 considered statistically significant. IOP and OCT values were presented as means and standard deviations (SD) in parentheses. Proportion of patients with IOP $\geq 25$, proportion of patients with OCT CFT $\geq 350$ microns, and proportion with $\mathrm{CME}$ on qualitative $\mathrm{OCT}$ analysis were also compared using chi-square analysis.

\section{Results}

\section{Patient Demographics}

Data were collected from 23 eyes of 23 patients undergoing PPV with air/gas tamponade in each group. Demographic information, preoperative diagnosis, and surgical procedures are summarized in Table 1. Overall, there were more men than women in each group (52\% vs. $48 \%$ in the test group, $57 \%$ vs. $43 \%$ in the control group), and the mean age was 69 years in the test group and 66 years in the control group. The majority of patients were Caucasian in each group, but there was a similar ethnic distribution in both groups. The most common diagnosis was epiretinal membrane ( $48 \%$ in each group), with $13 \%$ macular hole, 13\% rhegmatogenous retinal detachment, $13 \%$ vitreous hemorrhage, $9 \%$ retained silicone oil after complex retinal detachment repair, and $4 \%$ vitreomacular traction.

\section{Primary Outcome}

The proportion of eyes with complete ACCC at postoperative day 1, 7, and 30 are presented in Table 2 . Overall, there was a statistically-significant difference in the test group compared to the control group in ACCC at
Table I Baseline Demographics, Diagnoses, and Surgical Procedures for Study Subjects

\begin{tabular}{|c|c|c|}
\hline & $\begin{array}{l}\text { Study } \\
\text { Group } \\
(n=23)\end{array}$ & $\begin{array}{r}\text { Control } \\
\text { Group } \\
(n=23)\end{array}$ \\
\hline Age (years), mean (SD) & $69.0(10.4)$ & $66.4(9.3)$ \\
\hline \multicolumn{3}{|l|}{ Gender, n (\%) } \\
\hline Male & $12(52)$ & $13(57)$ \\
\hline Female & II (48) & $10(43)$ \\
\hline \multicolumn{3}{|l|}{ Ethnicity } \\
\hline Caucasian & $17(74)$ & $16(70)$ \\
\hline Hispanic or Latino & $3(13)$ & $3(13)$ \\
\hline African American & $2(9)$ & $3(13)$ \\
\hline Asian & I (4) & $2(9)$ \\
\hline \multicolumn{3}{|l|}{ Surgical Diagnosis, n (\%) } \\
\hline Epiretinal Membrane & II (48) & II (48) \\
\hline Macular Hole & $3(13)$ & $3(13)$ \\
\hline Retinal Detachment & $3(13)$ & $3(13)$ \\
\hline Vitreous Hemorrhage & $3(13)$ & $3(13)$ \\
\hline Retained Silicone Oil & $2(9)$ & $2(9)$ \\
\hline Vitreomacular Traction & I (4) & I (4) \\
\hline \multicolumn{3}{|l|}{ Procedure Performed, n (\%) } \\
\hline Pars plana vitrectomy & $18(78)$ & $18(78)$ \\
\hline Combined phaco-vitrectomy & $5(22)$ & $5(22)$ \\
\hline
\end{tabular}

Notes: Study Group refers to the Dexamethasone Intracanalicular Insert (DII) Group and Control Group refers to the Standard Topical Steroid Therapy Group. Abbreviation: SD, standard deviation.

postoperative day $7 ; 65 \%$ of patients in the dexamethasone insert group achieved complete ACCC as compared to $35 \%$ in the topical steroid group $(\mathrm{p}=0.01)$.

\section{Secondary Outcomes}

Secondary outcomes of IOP, vision, and OCT are summarized in Table 2. In the dexamethasone intracanalicular insert group, mean IOP increased from $12.9 \mathrm{mmHg}$ at baseline to $13.1 \mathrm{mmHg}$ at Day 30, and no eyes had increase in IOP greater than $25 \mathrm{mmHg}$ during the observation period. In the topical steroid group, mean IOP decreased from $15.3 \mathrm{mmHg}$ at baseline to $13.6 \mathrm{mmHg}$ at Day 30, but two eyes had increase in IOP to greater than $25 \mathrm{mmHg}$ during the observation period; both required topical glaucoma medications to lower the IOP.

The dexamethasone insert group had improvement in mean CFT on OCT from 390 microns at baseline to 293 microns at Day 30 (mean decrease 97 microns; similarly, the topical steroid group had improvement from 356 microns at baseline to 297 microns at Day 30 (Table 2). 
Table 2 Primary and Secondary Outcomes for Study Subjects

\begin{tabular}{|l|r|r|}
\hline & Study Group (n=23) & Control Group (n=23) \\
\hline Anterior Chamber Cell Clearance & & $2(9 \%)$ \\
Day I \# (\%) & $3(13 \%)$ & $8(35 \%)$ \\
Day 7 \# (\%) & $15(65 \%)$ & $0(0 \%)$ \\
Day 30 \# (\%) & $0(0 \%)$ & $0.0 I^{*}$ \\
\hline IOP, mmHg & & $15.3(9-22)$ \\
Baseline Mean (Range) & $12.9(5-20)$ & $10.7(5-32)$ \\
Day 7 Mean (Range) & $10.5(5-18)$ & $13.6(6-23)$ \\
Day 30 Mean (Range) & $13.1(5-23)$ & $-1.7(-5$ to +10$)$ \\
Mean $\triangle$ From Baseline (Range) & $0.2(-4$ to +8$)$ & $2(9 \%)$ \\
IOP >25 mmHg \# (\%) & $0(0 \%)$ & 0.3 \\
\hline OCT CFT, microns & & $356.0(250-527)$ \\
Baseline Mean (Range) & $390.0(237-64 I)$ & $296.8(220-412)$ \\
Day 30 Mean (Range) & $292.5(220-405)$ & $-49.5(-140$ to +92) \\
Mean Change From Baseline & $-97.5(-289$ to +9) & $3(13 \%)$ \\
Cystoid Macular Edema \# (\%) & $1(4 \%)$ & 0.3 \\
\hline
\end{tabular}

Notes: Study Group refers to the Dexamethasone Intracanalicular Insert (DII) Group and Control Group refers to the Standard Topical Steroid Therapy Group; ${ }^{a}$ chi-square analysis was employed to compare the groups; *Statistically significant $(p \leq 0.05)$.

Abbreviations: \#, absolute number of subjects; \%, percent of subjects in group; IOP, intraocular pressure; mmHg, millimeters of mercury; $\Delta$, change; OCT CFT, optical coherence tomography central foveal thickness.

Furthermore, one eye in the dexamethasone insert group had cystoid macular edema on OCT at Day 30 compared to 3 in the topical steroid group (not statistically significant, $\mathrm{p}=0.3$ ).

The dexamethasone insert was well tolerated in all eyes. No other adverse events beyond those described above were observed.

Seven out of seven (100\%) patients having undergone previous vitrectomy surgery in the contralateral eye preferred the dropless regimen with the dexamethasone intracanalicular implant compared with the traditional topical steroid therapy regimen for convenience and comfort.

\section{Discussion}

Control of postoperative inflammation with topical corticosteroid therapy is a cornerstone in postoperative management after PPV. This is the first report investigating the safety and efficacy of the dexamethasone DII for sustained-release delivery of corticosteroid therapy following PPV.

This study demonstrated that the DII provided statistically-significant higher rates of complete ACCC as compared to traditional topical corticosteroid therapy at postoperative day $7.65 \%$ of the DII group had complete ACCC at day 7 as compared to $35 \%$ in the topical steroid group $(\mathrm{p}=0.01)$.

Importantly, there were no safety signals in the DII group, namely no significant rise inIOP, no increase in the rate of cystoid macular edema (CME), and no migration of the implant in the postoperative period. In fact, there were trends towards lower incidence of IOP elevation greater than $25 \mathrm{mmHg}$ and decrease in the incidence of CME at Day 30 in the DII group compared to the topical steroid group, although the differences were not statistically significant.

The DII also offers several significant safety advantages over other available sustained-release corticosteroid platforms. Options for sustained-delivery steroid after vitrectomy include sub-Tenon's ${ }^{11}$ or intravitreal ${ }^{12}$ injection of triamcinolone acetonide on-label (Triesence: Alcon, Dallas, TX) or off-label (Kenalog-40: Bristol-Myers Squibb, Princeton, NJ), and dexamethasone intravitreal implant $^{13}$ (Ozurdex: Allergan, Irvine, CA). While subTenon's injection of steroids is an attractive approach in that it is not intraocular, there are potential issues with the predictability of dosing via this route, risk of IOP rise, local tissue toxicity, risk of intravascular injection, and unpredictable duration of effect. ${ }^{9}$ Intravitreal approaches are attractive in that they deliver high effective dosage locally, but there are potential associated risks such as retinal toxicity, ${ }^{14}$ retinal detachment, and endophthalmitis. ${ }^{12}$ There are also added risks of implant migration in pseudophakic eyes with the dexamethasone intravitreal implant, ${ }^{15}$ and dispersion of the steroid suspension resulting in a "snowglobe effect" with intravitreal 
agents. These toxicity and migration risks may be greater in air-, gas-, or oil-filled eyes.

An anterior chamber intraocular suspension of dexamethasone has also been approved for postoperative inflammation control following intraocular surgery (Dexycu ${ }^{\circledR}$ EyePoint Pharmaceuticals, Inc., Watertown, MA). ${ }^{16,17}$ This product requires intraocular injection into the anterior chamber at the conclusion of surgery and has a $66 \%$ rate of ACCC at postoperative day 14 . However, the IOP elevation rate is approximately 4-fold higher than topical therapy, likely attributable to higher intraocular concentrations of the drug resulting from direct intraocular administration. ${ }^{16-18}$ There is also potential of direct toxicity of the agent on the corneal endothelium if the patient is in a face-down position and of trauma to the lens in phakic patients during intraoperative administration.

Overall, sustained-release dexamethasone offers additional benefits beyond eliminating concerns with compliance. Quality of life improvement is derived by avoiding topical eye drops, as $96 \%$ of cataract surgery patients rated the insert as convenient/very convenient when contrasted with their past experience using topical ophthalmic medications. ${ }^{19}$ In this series, all seven patients undergoing eye surgery in the second eye preferred the dropless postoperative regimen in terms of convenience and comfort. Simplifying the regimen may also reduce patient callbacks to the office for issues related to postoperative medications. $^{8}$

The key strength of this study is the analysis of safety and efficacy of the dexamethasone intracanalicular insert in a previously unreported patient population, namely patients undergoing PPV. Also, the inclusion of data from consecutive patients from a single surgeon minimizes the potential for selection bias associated with retrospective studies. As with all studies, the current study has limitations including the small sample size, the variety of procedures, and the retrospective design of the study.

\section{Conclusion}

This small, retrospective, consecutive case study demonstrated that the DII provided excellent control of postoperative inflammation following PPV with air/gas tamponade without significant safety issues. This regimen should be considered for patients undergoing PPV to safely and effectively control postoperative inflammation, to ensure patient compliance, and to improve patient satisfaction.

\section{Ethics Approval}

The current study was approved by the Western Institutional Review Board. Due to the retrospective nature of the study, patient consent to review the data was waived by the Western IRB. The data was de-identified after collection to protect patient confidentiality and privacy. The study adhered to the principles of the Declaration of Helsinki.

\section{Acknowledgments}

Editorial support in the development of this manuscript was provided by Tony Realini, MD and funded by Ocular Therapeutix, Inc. in accordance with Good Publication Practice (GPP3) guidelines. Presented at the 2020 ASRS Annual Meeting, 2020 Retina Society Annual Meeting, and 2020 Euretina Meeting.

\section{Author Contributions}

The authors contributed to this work in the following ways:

1. Made a significant contribution to the work reported, whether that is in the conception, study design, execution, acquisition of data, analysis and interpretation, or in all these areas.

2. Have drafted or written, or substantially revised or critically reviewed the article.

3. Have agreed on the journal to which the article will be submitted.

4. Reviewed and agreed on all versions of the article before submission, during revision, the final version accepted for publication, and any significant changes introduced at the proofing stage.

5. Agree to take responsibility and be accountable for the contents of the article.

\section{Funding}

The study was supported in part by an unrestricted research grant from Ocular Therapeutix, Inc. The supporting source had no involvement in study design, collection, analysis, and interpretation of data.

\section{Disclosure}

Dr. Suñer is a consultant for Ocular Therapeutix, Alcon, Alimera, Allergan, Genentech, Novartis, and Regeneron. Dr. Peden is a consultant for Genentech. The authors report no other conflicts of interest in this work. 


\section{References}

1. Machemer R, Benson WE. Vitrectomy: A Pars Plana Approach. Grune \& Stratton; 1975.

2. Fujii GY, De Juan E, Humayun MS, et al. A new 25-gauge instrument system for transconjunctival sutureless vitrectomy surgery. Ophthalmology. 2002;109(10):1807-1812. doi:10.1016/s01616420(02)01179-x

3. Oshima Y, Wakabayashi T, Sato T, Ohji M, Tano Y. A 27-gauge instrument system for transconjunctival sutureless microincision vitrectomy surgery. Ophthalmology. 2010;117(1):93-102.e2. doi:10.1016/j.ophtha.2009.06.043

4. Inoue Y, Kadonosono K, Yamakawa T, et al. Surgically-induced inflammation with 20-, 23-, and 25-gauge vitrectomy systems: an experimental study. Retina Phila Pa. 2009;29(4):477-480. doi:10.1097/IAE.0b013e31819a6004

5. An JA, Kasner O, Samek DA, Lévesque V. Evaluation of eyedrop administration by inexperienced patients after cataract surgery. $J$ Cataract Refract Surg. 2014;40(11):1857-1861. doi:10.1016/j. jcrs.2014.02.037

6. Hennessy AL, Katz J, Covert D, et al. A video study of drop instillation in both glaucoma and retina patients with visual impairment. Am J Ophthalmol. 2011;152(6):982-988. doi:10.1016/j. ajo.2011.05.015

7. Hermann MM, Ustündag C, Diestelhorst M. Electronic compliance monitoring of topical treatment after ophthalmic surgery. Int Ophthalmol. 2010;30(4):385-390. doi:10.1007/s10792-010-9362-3

8. Newman-Casey PA, Robin AL, Blachley T, et al. The most common barriers to glaucoma medication adherence: a cross-sectional survey. Ophthalmology. 2015;122(7):1308-1316. doi:10.1016/j.ophtha.20 15.03.026

9. Walters T, Bafna S. Efficacy and safety of sustained release dexamethasone for the treatment of ocular pain and inflammation after cataract surgery: results from two phase 3 studies. J Clin Exp Ophthalmol. 2016;7(4):1-11. doi:10.4172/2155-9570.1000572

10. Tyson SL, Bafna S, Gira JP, et al. Multicenter randomized phase 3 study of a sustained-release intracanalicular dexamethasone insert for treatment of ocular inflammation and pain after cataract surgery. $J$ Cataract Refract Surg. 2019;45(2):204-212. doi:10.1016/j. jcrs.2018.09.023
11. Kim SY, Shin JA, Yum HR, Han H, Yang JW, Park YH. Long-term trends in intraocular pressure after combined vitrectomy with sub-tenon injection of triamcinolone acetonide. Retina. 2015;35 (3):564-569. doi:10.1097/IAE.0000000000000333

12. Lai CC, Wang NK, Wu WC, et al. The long-term anatomical and visual effect of intravitreal triamcinolone injection during vitrectomy for the treatment of idiopathic macular epiretinal membrane. Cutan Ocul Toxicol. 2011;30(4):292-297. doi:10.3109/15569527.20 11.568031

13. Zheng A, Chin EK, Almeida DR, et al. Combined vitrectomy and intravitreal dexamethasone (ozurdex) sustained-release implant. Retina. 2016;36:2087. doi:10.1097/IAE.0000000000001063

14. Sisk RA, Patel YP, Foster RE, Petersen MR. Erosive retinopathy and retinal detachment from depot Intravitreal triamcinolone acetonide injection at the end of pars plana vitrectomy. Ophthalmic Surg Lasers Imaging Retina. 2019;50(10):613-619. doi:10.3928/2325816020191009-03

15. Rahimy E, Khurana RN. Anterior segment migration of dexamethasone implant: risk factors, complications, and management. Curr Opin Ophthalmol. 2017;28(3):246-251. doi:10.1097/ICU.000000000 0000365

16. Grzybowski A, Brockmann T, Kanclerz P, Pleyer U. Dexamethasone intraocular suspension: a long-acting therapeutic for treating inflammation associated with cataract surgery. $J$ Ocul Pharmacol Ther. 2019;35(10):525-534. doi:10.1089/jop.2019.0072

17. Donnenfeld E, Holland E. Dexamethasone intracameral drug-delivery suspension for inflammation associated with cataract surgery: a randomized, placebo-controlled, Phase III trial. Ophthalmology. 2018;125(6):799-806. doi:10.1016/j.ophtha.2017.12.029

18. Lindstrom RL, Galloway MS, Grzybowski A, Liegner JT. Dropless cataract surgery: an overview. Curr Pharm Des. 2017;23(4):558-564. doi: $10.2174 / 1381612822666161129150628$

19. Gira JP, Sampson R, Silverstein SM, Walters TR, Metzinger JL, Talamo JH. Evaluating the patient experience after implantation of a $0.4 \mathrm{mg}$ sustained release dexamethasone intracanalicular insert (Dextenza ${ }^{\mathrm{TM}}$ ): results of a qualitative survey. Patient Prefer Adherence. 2017;11:487-494. doi:10.2147/PPA.S126283
Clinical Ophthalmology

\section{Publish your work in this journal}

Clinical Ophthalmology is an international, peer-reviewed journal covering all subspecialties within ophthalmology. Key topics include: Optometry; Visual science; Pharmacology and drug therapy in eye diseases; Basic Sciences; Primary and Secondary eye care; Patient Safety and Quality of Care Improvements. This journal is indexed on PubMed
Central and CAS, and is the official journal of The Society of Clinical Ophthalmology (SCO). The manuscript management system is completely online and includes a very quick and fair peer-review system, which is all easy to use. Visit http://www.dovepress.com/ testimonials.php to read real quotes from published authors.

\section{Dovepress}

